\title{
Development and evaluation of the EOL-ICDQ as a measure of experiences, attitudes and knowledge in end-of-life in patients living with an implantable cardioverter defibrillator
}

\author{
Ingela Thylén, Marika Wenemark, Christina Fluur, Anna Strömberg, Kärstin Bolse and \\ Kristofer Årestedt
}

\author{
Linköping University Post Print
}

\section{Tweet}

N.B.: When citing this work, cite the original article.

Original Publication:

Ingela Thylén, Marika Wenemark, Christina Fluur, Anna Strömberg, Kärstin Bolse and Kristofer Arestedt, Development and evaluation of the EOL-ICDQ as a measure of experiences, attitudes and knowledge in end-of-life in patients living with an implantable cardioverter defibrillator, 2014, European Journal of Cardiovascular Nursing, (13), 2, 142151.

http://dx.doi.org/10.1177/1474515113515563

Copyright: Elsevier / SAGE Publications (UK and US): 12 month Embargo http://www.uk.sagepub.com/home.nav

Postprint available at: Linköping University Electronic Press http://urn.kb.se/resolve?urn=urn:nbn:se:liu:diva-105740 


\section{Development and evaluation of the "EOL-ICD Questionnaire" as a measure of Experiences, Attitudes and Knowledge in End-of-Life in patients living with an Implantable Cardioverter Defibrillator}

Thylén Ingela, RN, PhD, Ass Prof ${ }^{1}$, Wenemark Marika, Statistician, PhD ${ }^{2}$, Fluur Christina, $\mathrm{MD}^{3}$, Strömberg Anna, RN, PhD, FAAN, Prof ${ }^{1}$, Bolse Kärstin, RN, PhD, Ass Prof ${ }^{4}$, and Årestedt Kristofer, RN, PhD, Ass Prof ${ }^{5}$

${ }^{1}$ Division of Nursing Sciences, Department of Medicine and Health Sciences, Faculty of Health Sciences, Linköping University, Department of Cardiology, County Council of Östergötland, Linköping, Sweden, e-mail: anna.stromberg@liu.se

${ }^{2}$ Division of Community Medicine, Department of Medicine and Health Sciences, Faculty of Health Sciences, Linköping University and Centre for Public Health, County Council of Östergötland, Linköping, Sweden, e-mail: marika.wenemark@liu.se

${ }^{3}$ Department of Cardiology, County Council of Östergötland, Linköping, Sweden, e-mail: christina.fluur@lio.se

${ }^{4}$ School of Social and Health Sciences, Halmstad University, Sweden, e-mail: karstin.bolse@hh.se

${ }^{5}$ School of Health and Caring Sciences, Faculty of Health, Social Work and Behavioral Sciences, Linnaeus University, Kalmar, Sweden, and Division of Nursing Sciences, Department of Medicine and Health Sciences, Faculty of Health Sciences, Linköping University, Sweden, e-mail: kristofer.arestedt@liu.se

\section{Address for Correspondence}

Ingela Thylén, Ass Prof., Department of Cardiology, Linköping University Hospital, 58185 Linköping, Sweden, Tel: + 461010300 00, Fax: +46 1010322 24, E-mail:

ingela.thylen@lio.se

\section{Funding}

This study was funded with grants from the Medical Research Council of Southeast Sweden (FORSS), the Swedish Heart- and Lung Foundation, and Linköping University Hospital Research Fund, Sweden.

\section{Word count 3731}




\section{ABSTRACT}

Background Due to extended indications and resynchronization therapy, many implantable cardioverter defibrillator (ICD) recipients will experience progressive comorbid conditions and are likely to die of other causes than cardiac death. It is therefore even more important to elucidate the ICD patients' preferences when nearing end-of-life. Instead of avoiding the subject of end-of-life, a validated questionnaire may be helpful to not only explore patients' experiences and attitudes about end-of-life concerns but also investigate how knowledgeable they are about the function of the ICD in end-of-life. Validated instruments testing patients' knowledge concerning end-of-life issues are still scarce.

Aim To develop and evaluate the measurement properties of the "Experiences, Attitudes and Knowledge of End-of-Life issues in Implantable Cardioverter Defibrillator patients; the EOL-ICD Questionnaire".

Methods The instrument was tested for validity, respondent satisfaction, and for homogeneity and stability in the Swedish language. The EOL-ICD Questionnaire was also validated, but not pilot tested, into an English version.

Results The final instrument contained three domains, which were clustered into 39 items; measuring experiences (10 items), attitudes (18 items) and knowledge (11 items) of end-oflife concerns in ICD patients. In addition, the questionnaire also contained items on sociodemographic background (6 items) and ICD-specific background (8 items) that can be used together with the main questionnaire. The validity and reliability properties were considered sufficient.

Conclusions The EOL-ICD Questionnaire has the potential to be used in clinical practice and future research. Further studies are needed using this instrument in an Anglo-Saxon context with a sample of English speaking ICD-recipients. 
Keywords Cognitive interviewing; EOL-ICD Questionnaire; Implantable Cardioverter Defibrillator; Proactive communication; Psychometric testing; Respondent satisfaction 


\section{INTRODUCTION}

Since over twenty years implantable cardioverter defibrillators (ICDs) have been the treatment for choice for patients at risk for life-threatening arrhythmias. When combined with resynchronisation therapy (CRT) in heart failure patients, it improves symptoms and survival. ${ }^{1}$ This implies that the ICD-population will be older and many ICDrecipients experience progressive morbidity and are likely to die of chronic illness. Seen from this perspective, it is important to elucidate the ICD-patients' knowledge and preferences about deactivation when nearing end-of-life. There are several obstacles to achieve effective and valuable patient education. Firstly, many professionals still feel uncomfortable discussing end-of-life issues with their patients ${ }^{2,3}$ and lack competence and skills in how to communicate this issue. ${ }^{4}$ Secondly, professionals may feel uncertain about if and/or when patients prefer to discuss generator replacement and deactivation, which could result in a delayed end-of-life discussion. ${ }^{5}$ Therefore, a validated questionnaire may be helpful to explore patients' experiences and attitudes about end-of-life concerns and investigate how knowledgeable they are about the function of the ICD in end-of-life. A validated questionnaire could also provide the professionals with information about how and when to discuss these issues with a particular patient. Moreover, such a questionnaire may be useful as a communication tool helping the patient to reflect on end-of-life issues.

Many health instruments have been constructed with focus on what professionals and researchers want to measure, whereas patients have not been seen as important cooperation partners in the construction process. ${ }^{6}$ From the professionals' standpoint, an instrument needs to have good measurement properties, being easy to use and functional for clinical practice. However, over the years, it has become increasingly difficult to achieve high participation in surveys, ${ }^{7}$ and it is therefore important to satisfy needs and expectations also from patients. In order to be a trustworthy instrument the questions need 
to be valid and relevant also from patients' point of view, and the questionnaire used in a way that patients feel secure to give truthful and honest answers. ${ }^{8}$

Validated instruments testing patients' attitudes concerning end-of-life issues are rare, but Conelius ${ }^{9}$ has recently developed a survey aiming to determine ICD-recipients' preferences, barriers, and understanding towards an advanced directive. Also Kirkpatrick et al. ${ }^{10}$ has developed a questionnaire focusing on advanced directives; including living wills and power of attorney for healthcare, but this survey was primarily based on expert validation. Others have used scenarios and purpose-design questions to ascertain patients' knowledge about deactivation ${ }^{11}$ and their opinions regarding withdrawal of therapy at the end-of-life. ${ }^{11-13}$ Finally, Herman et al. ${ }^{14}$ reported a questionnaire about expected ICD benefits, feelings and circumstances under which the ICD-recipient would want to deactivate the ICD. Even if one of the existing instruments has been validated in terms of factor structure and test-retest reliability, ${ }^{9}$ none have included the patients' perspectives during the whole construction process. To our knowledge, no instrument aiming at measuring both 1) experiences and preferences about when and how discussions about ICD at end-of-life should be raised, 2) attitudes to deactivation and withdrawal of therapy and, 3) knowledge about ICD-therapy at end-of-life, exists.

\footnotetext{
Aim

To develop and evaluate respondent satisfaction and measurement properties of the “Experiences, Attitudes and Knowledge of End-of-Life Issues in Implantable Cardioverter Defibrillator Patients (EOL-ICDQ) Questionnaire”.
} 


\section{METHODS}

\section{Design and setting}

This study had a methodological design where the EOL-ICDQ was developed and evaluated in a Swedish setting. The construction of the questionnaire involved three phases and continued during 2010-2012 (Figure 1). Finally, a translation/back-translation from Swedish to English was performed.

\section{Participants}

The participants were recruited if they had ICDs for $\geq 6$ months and were not in the palliative phase of a terminal illness. They were identified from medical records and booking system of five Swedish hospitals (pilot) and from the Swedish ICD- and Pacemaker registry (test-retest). To achieve maximal variation in the samples ${ }^{15}$ quota sampling was performed to ensure broad ranges of background characteristics that were considered important, including age, gender, marital status, education, etiology, time since first implantation, prior generator replacement, and prior experience with defibrillator shocks. A letter with written information about the study and an invitation to participate was sent to representative patients. After completing the informed consent, the respondents were mailed the questionnaire after 2-3 weeks. The participants in the pilot were asked to complete a related survey evaluating respondent satisfaction with the questionnaire. The test-retest participants were in their part instructed to complete the questionnaire twice, with the second questionnaire being mailed out 2-3 weeks after completion of the first one. The participants were informed in writing about the purpose and the structure of the study before they gave their informed consent. Participation was voluntarily and the participants were informed that they could withdraw from the study at any time. The investigation were approved by the Regional Ethics Committee for Human Research at the University of 
Linköping, Sweden (D-nr 2010/321-31 and D-nr 2011/434-31) and conformed with the principles outlined in the Declaration of Helsinki. ${ }^{16}$

\section{Questionnaire development and evaluation}

\section{Phase I}

The content in this self-administrated questionnaire was based on extensive literature reviews and the results from a qualitative study that has been previously published. ${ }^{17}$ The themes emerging from the literature review included (a) patients' experiences of discussing deactivation with healthcare professionals, and (b) patients' attitudes of deactivation. The qualitative study additionally revealed themes about (c) patients' knowledge/understanding of the ICD in an end-of-life setting. A pool of items and a definition of each of the domains included in the questionnaire were initially drafted. Thereafter the investigators, a group of five interdisciplinary clinicians and clinical researchers (nurses and physicians) specialized in ICD-care, one statistician skilled in survey design and one ICD-recipient met several times to discuss the layout, content, and response alternatives in the preliminary questionnaire. The discussions resulted in the definition of attitudes about end-of-life also ought to include not only deactivation but also withdrawal of therapy at battery end-ofservice, and adding items not only about discussions with the professionals but also with the family.

The items in the questionnaire were written as clear, brief statements with two or three response alternatives and divided into three domains; Experiences about end-of-life issues, Attitudes about discussions and actions, and Knowledge about the ICD in relation to end-of-life. The preliminary questionnaire consisted of 37 items. In addition, optional items on socio-demographic background (6 items) and ICD-specific background (8 items) were also developed in order to be used together with the main questionnaire where appropriate. 
The preliminary questionnaire and the background questions were given to seven clinical experts within nursing and cardiology to be reviewed and commented. Based on their suggestions, one new knowledge item was added and three items were reworded due to misinterpretation of the content. The expert validation was then considered having a good face- and content validity.

\section{Phase II}

To evaluate and improve respondent satisfaction of the questionnaire, 32 patients (19 men and 13 women, median age 63, range 33-85) were recruited for (1) completing the questionnaire and, (2) completing a separate evaluation survey. They were instructed to validate each item in the questionnaire regarding relevance, clearness and easiness to read and complete, as suggested by Polit and Beck ${ }^{18}$, for example; "Is the question relevant for you as an ICD-recipient?"; “Can the question be interpreted in several ways?”; "Is the reply alternatives in the question appropriate for you to give your answer?"; and "Is the question perceived as too sensitive?". In order to gain a deeper understanding of the respondent satisfaction, cognitive interviews were performed by telephone with three participants using "Thinking loud technique". ${ }^{19}$ The patients were sent the questionnaire in advance, but asked to fill it in during the interview. They were invited to comment on the questions with thoughts that came up in their head when they answered the questionnaire. Probing questions were used to clarify or explore a previous response that came up during the interview.

The questionnaire was judged by the participants to be relevant, user-friendly and having sufficient clarity and readability, and they found it relevant to complete. None of the participants considered any of the questions as too sensitive to answer, but two questions needed to be rephrased after the cognitive interviews. One new attitude item was added 
about when the patient would like professionals to broach the question of what it involves to deactivate the ICD since it had been noted that the existing choices were not sufficient to cover all aspects. In addition to this, one new experience item regarding philosophy of life was added. This modification was based on the comments during the interviews as being important in this setting. After these adjustments, again one patient validated the questionnaire without having any new comments on the content or the reply alternatives in the questionnaire.

\section{Phase III}

The final questionnaire was evaluated regarding measurement properties. To test reproducibility, 109 patients (Table 1) were asked to complete the final questionnaire twice, with a time interval of 2-3 weeks. The final version contained three domains measuring Experiences of end-of-life issues (10 items), Attitudes about discussions and actions (18 items) and Knowledge about the ICD in relation to end-of-life (11 items, divided into ethical aspects, function of the ICD, and practical consequences). The respondents list their answer in an unforced-choice format as "Yes/No" or "Can't take a stand”, “Agree/Don't agree", “True/False", or "Don't know”. Items belonging to the Experience and Attitude domains are used as single items while items belonging to the Knowledge scale are used as sum scores. The optional questions on socio-demographic background include items on age and gender, ethnicity, marital- and occupational status, and educational level (6 items). Items regarding ICD-specific background are; time since implantation, cardiac resynchronization therapy, primary or secondary prevention therapy, generator replacement, perceived general health and general satisfaction with the ICD, experience of shock therapy, and rating the severity of the shock induced pain and/or anxiety (8 items). 


\section{Statistical analyses}

Descriptive statistics were used to describe the distribution of item responses and pattern of missing data. The reproducibility (test-retest reliability) was evaluated using kappa statistics. An un-weighted kappa was calculated as the items consisted of dichotomous or not ordered response categories. The agreement according to the kappa statistics were interpreted as followed; poor $<0.20$, Fair 0.20-0.40, Moderate 0.40-0.60, Good $0.60-0.80$, and Very good $0.80-1.00 .^{20}$ As the knowledge domain use the total score as a measure of knowledge, true answers were coded as 1 while errors and the alternative "I don't know" was coded as 0 . The score for the eleven items was thereafter added in a total score. The Kuder-Richardson formula 20 (KR20) was calculated to evaluate internal consistency of the items in the knowledge domain. A sufficient level of KR20 was set to $\geq 0.70$. In addition, the items in the knowledge domain were also evaluated regarding difficult level and discrimination ability. ${ }^{21}$ To evaluate the difficulty level of each item, the item difficulty index was calculated. This index represents the proportion of participants who answered the item correctly, i.e. higher values imply easier items. To evaluate the discrimination ability, item discrimination coefficients were calculated. For this purpose, the point biserial correlation $\left(\mathrm{r}_{\mathrm{pb}}\right)$ between the performance on the individual item and the overall test was used. Stronger correlations imply better discrimination ability. A sufficient level of the item discrimination index was set to $r_{p b} \geq 0.30$.

\section{Translational procedure}

The questionnaire was translated into an American English version through a translation/back-translation procedure. ${ }^{22,23}$ First, a native English speaker fluent in both languages translated the questionnaire into English, and then a native Swedish speaker fluent in both languages re-translated it into Swedish, with no previous knowledge of the 
original survey. Differences between the English version and the re-translated Swedish version, as well as possible transcultural differences, were discussed with all investigators

in order to improve the quality of the English translation. ${ }^{22,23}$ Finally, the English version of the EOL-ICDQ were linguistically tested and expert validated with face- and content validity with three native English speaking researchers in the US familiar within the ICD area. The questionnaire was revised with minor changes in respect to language and cultural differences. The English version was considered having a sound content validity and being translational and culturally equivalent to the Swedish version.

\section{RESULTS}

\section{Measurement properties}

\section{Experience of end-of-life issues}

The number of missing data was low $(<1 \%)$ and concentrated to three of the items; "I have discussed the illness course for my heart disease with my doctor" (item 7), "I have a religious faith/outlook on life" (item 9), and "I often think about questions concerning end-of-life" (item 10). The items "I have told my next-of-kin my wishes regarding the shocks in my ICD, if I become seriously ill with some fatal disease" (item 5) and "I have at some point considered asking my doctor to turn off the shocks in my ICD” (item 6) was most skewed. Most items demonstrated good (item 1,2,7,8,9,10), or moderate reproducibility (item 4,5). Fair reproducibility was showed for two items; "I have discussed with my doctor what turning off the shocks involves" (item 3) and "I have at some point considered asking my doctor to turn off the shocks in my ICD” (item 6). No item showed poor reproducibility (Table 2 ). 


\section{Attitudes about discussions and actions}

In the Attitudes about discussions, missing data was found for all items except "Towards end-of-life, during the last days" (item 10). The missing values were equally distributed across the items with 1-3 missing each (1.5\%). The item "I myself will raise the question when I feel the need to" (item 2) was most skewed distributed. Most items had a moderate (items 4,5,7,8,9) or fair (items 1,3,6,10) reproducibility. One item demonstrated poor reproducibility; "I myself will raise the question when I feel the need to" (item 2) (Table 3).

In the Attitudes about actions, missing data was demonstrated in five out of eight items (1\%). The frequency of missing data was low but still most common for the item "At the end of my life, I want my next-of-kin to decide if the shocks are to be turned off" (item 18). Regarding the distribution of scores between the Yes and No alternatives, little variation was showed for the items; "I want to have the battery in my ICD replaced even if I have not received any shocks" (item 11), "I want to have the battery in my ICD replaced even if I have reached an advanced age (item 13), and "At the end-of-life, I myself want to decide if the shocks are to be turned off or not (item 16). The items showed a moderate (items $13,14,17$ ) or fair (items $11,12,15,16,18$ ) reproducibility. No item showed poor reproducibility (Table 4$)$.

\section{The Knowledge scale}

Only one item in the knowledge scale had missing data, "In order to turn off the shocks in an ICD, the ICD must be removed by surgery" (item 1). According to KR20, the internal consistency was 0.697 , close to the acceptable level of $\geq 0.70$. Except for one item, the distribution of the scores showed that most participants scored the True or False alternative. A large share of the respondents scored Don't know on the item "An ICD 
always delivers shocks in connection with end-of-life (item 9). This response alternative was also common for the item "A magnet can be used to temporarily turn off the ICD's shocks" (item 6). The item difficulty index (IDI) showed a satisfactory variation, the easiest item were "When an ICD's shocks are turned off, the heart stops beating (item 3, IDI=0.88) while the most difficult item were "An ICD always delivers shocks in connection with endof-life (item 9, IDI=0.30). All items demonstrated acceptable discrimination ability according to the critical level of the item discrimination coefficient $\left(\mathrm{r}_{\mathrm{pb}}>0.30\right)$ (Table 5).

\section{Experiences, Attitudes, and Knowledge in relation to end-of-life}

Overall, $40 \%$ had discussed the illness course with their physician. Eleven percent had discussed what turning off the shocks involves with someone in the ICD-team and 14\% had discussed the same topic with their family. Eleven percent had also told their next-ofkin their wishes regarding shock therapy if they should become seriously ill with some fatal disease. When it came to the participants attitudes on discussing deactivation and end-oflife issues, $29 \%$ were unwilling to have such a conversation, $61 \%$ stated that the question should be raised in connection with the ICD-implantation, and 70\% during the last days in life. The vast majority would like to replace the ICD at end-of-service, even if no shocks has been delivered (84\%), being seriously ill suffering from another disease (58\%), or has reached an advanced age (71\%). One third of the participants $(31 \%)$ was indecisive about deactivating the ICD or not if dying of cancer or other serious disease, and $46 \%$ was unable to take a stand about whether or not to keep the ICD active if receiving shocks daily. On the other hand, 26\% favoured an active ICD at end-of-life and $43 \%$ preferred deactivation under those circumstances. Finally, the participants were most knowledgeable about the statement; "When the shocks are turned off, the heart stops beating", with $83 \%$ correctly answering no. Least knowledge were found in; "When an ICD's battery voltage is 
beginning to wear, the ICD's functioning worsens", with $40 \%$ falsely answering yes. Most indecisive (48\%) was the participants about the falsely given statement; "An ICD always delivers shocks in connection with end-of-life".

\section{DISCUSSION}

The EOL-ICDQ was developed to identify patients' knowledge of the ICD in relation to end-of-life as well as their experiences and attitudes about such issues. To ensure the participants trust and confidence, efforts were made to involve patients in several steps during the construction and validation process. Validity was supported by the fact that the items were assessed to be adequate by the experts and patient in the research group and the participants in terms of the relevance and the focus of the study. The participants posed that the questionnaire was clear and easy to read and complete, and can thereby be considered to be a user-friendly questionnaire

The evaluation of the EOL-ICDQ showed that the items had sound measurement properties in terms of reproducibility. Although few items showed poor or fair reproducibility according to the kappa statistics, the absolute agreement was high. One reason for this discrepancy is that the kappa statistics takes the agreement occurring by chance into account. The kappa statistics can therefore be judge as a conservative test, in particular when the score distribution is highly skewed. This is for example reflected in "I myself will broach the question when I feel the need to" (item 2) which was most skewed distributed, had the highest absolute agreement, and lowest kappa value in the Attitudes about Discussions. However, it is possible that the reproducibility of the scale over time with a 2-3 week span was little too long. Ideally, this time frame should be 2-14 days. ${ }^{24}$

Also the knowledge scale showed sufficient measurement properties. The use of the response "Don't know" can probably explain the low number of missing data. Another 
strength using this response alternative is that it can reduce the number of persons who got a true answer by chance. To make it possible to calculate a total score for persons who used this alternative, an assumption is made that the person not have the knowledge, i.e., this alternative should be scored as an error answer. The large share of the persons who scored "Don't know" on item 6 and 9 may reflect that these items are too difficult or unclear. However, the item difficulty index showed that no item in the knowledge scale was too easy $(\geq 90)$ or difficult $(\leq 10)$. The great variation in item difficult level indicate that the knowledge scale have ability to discriminate between persons with different knowledge levels. This is also supported by the item discrimination coefficients, all item had a correlation $r_{p b} \geq 0.3 .^{20}$

One third of the participants were reluctant to discuss end-of-life issues, but according to existing expert consensus statements ${ }^{25,26}$ a discussion about deactivation should take place already in connection with the ICD-implantation. Further, one quarter preferred to keep the shocks in connection with end-of-life. This highlights the importance of being aware of the patient's unique preferences and needs. Since many of our participants were indecisive about their standpoint on deactivation, filling in the questionnaire in beforehand in the patient's home may possible ease reflection so that the ICD-recipient can be prepared and then serve as a base for a discussion with the ICD-team.

\section{Methodological issues}

It is a challenge to develop a questionnaire based on a scientific approach where it is possible to uncover sensitive existential issues. We are aware of the changeability of human experiences, attitudes and knowledge over time due to different life events and patients' illness trajectory and that this has implications for the understanding of the questionnaire and the 
application of it. However we think that EOL-Q is valid in a Swedish setting and will give a different and more humane understanding of these aspects in ICD-recipients.

The construct and testing of the EOL-ICDQ showed good psychometric properties. The findings provide satisfactory support for the content validity and respondent satisfaction as well as a good reproducibility support. The internal validity of this study was strengthened by the quota sampling to achieve maximal variation in the sample, being a representation of a larger population of patients with an ICD. Participants were not near their end-of-life. Most of the patients stated that they felt they were in "good" or "very good" health condition. This could have created a selection bias against patients who had many co-morbidities. However, when the participants self-reported their co-morbidities, several reported diabetes, lung problems, and kidney failure, while every tenth patient reported being suffering from cancer. This may imply that the questionnaire can be used in different stages of an illness trajectory.

\section{CONCLUSION}

In clinical practice, the EOL-ICDQ can be used as a communication tool and then documented in the medical records to assess experiences, attitudes, and knowledge in the individual ICD-recipient. It could be used as a reference point and an educational tool, following patients over time, to evaluate changes in their understanding or attitudes towards battery replacement and withdrawal of therapy. Further studies are however needed to test the instrument's usability as a communication in patients nearing end-of-life. Continued research with this questionnaire in clinical practice is important, since the focus addresses a clinically relevant concern that still is unexplored. Further studies are also needed testing this instrument in a sample of English speaking ICD-recipients. 
For access and permissions to use the questionnaire please email: ingela.thylen@lio.se 


\section{REFERENCES}

1. Epstein AE, Dimarco JP, Ellenbogen KA, et al, American College of Cardiology, American Heart Association Task Force on Practice Guidelines, American Association for Thoracic Surgery, Society of Thoracic Surgeons. ACC/AHA/HRS 2008 guidelines for device-based therapy of cardiac rhythm abnormalities. Heart Rhythm 2008; 5: 934955.

2. Goldstein NE, Mehta D, Siddiqui S, et al. "That's like crossing a bridge" complexities preventing physicians from discussing deactivation of implantable defibrillators at the end of life. J Gen Intern Med 2008; Suppl 1: 7-12.

3. Sherazi S, Daubert JP, Block RC, et al. Physicians' preferences and attitudes about endof-life care in patients with an implantable cardioverter-defibrillator. Mayo Clin Proc 2008; 83: 1139-1141.

4. Kelley AS, Mehta SS and Reid CM. Management of Patients with ICDs at the End of Life (EOL): A Qualitative Study. Am J Hosp Palliat Care 2009; 25: 440-446.

5. Russo JE. Deactivation of ICDs at the end of life: a systematic review of clinical practices and provider and patient attitudes. Am J Nurs 2011; 111: 26-35.

6. Streiner LS and Norman GR. Health measurement scales - a practical guide to their development and use. $4^{\text {th }}$ ed. Oxford: Oxford University Press, 2003, pp. 103-129.

7. de Leeuw E, de Heer W. Trends in household survey nonresponse: a longitudinal and international comparison. In: Survey Non-response. Groves RM, Dillman DA, Eltinge JL, Little RJA (eds.). New York: Wiley 2002, pp. 41-54.

8. Wenemark M. The respondent's perspective in health-related surveys. The role of motivation. Linköping University Medical dissertations No. 1193, LiU Tryck, Linköping, Sweden, 2010. 
9. Conelius J. The development, refinement, and psychometric testing of the Attitude Toward Advanced Directive Survey in Implantable Cardioverter Defibrillator Patients. J Cardiovasc Nurs 2013; 28: 238-244.

10. Kirkpatrick JN, Gottlieb M, Sehgal P, et al. Deactivation of Implantable Cardioverter Defibrillators in Terminal Illness and End of Life Care. Am J Cardiol 2012; 109: 91-94.

11. Pedersen SS, Chaitsing R, Szili-Torok T, et al. Patients' perspective on deactivation of the implantable cardioverter-defibrillator near the end of life. Am J Cardiol Epub ahead of print 10 Mar 2013. DOI: 10.1016/j.amjcard.2013.01.296.

12. Kapa S, Mueller PS, Hayes DL, et al. Perspectives on withdrawing pacemaker and Implantable Cardioverter Defibrillator therapies at end of life: results of a survey of medical and legal professionals and patients. Mayo Clin Proc 2010; 85: 981-990.

13. Dodson JA, Fried TR, van Ness PH, Goldstein NE, Lampert R. Patient preferences for deactivation of Implantable Cardioverter-Defibrillators. JAMA Intern Med $2013 ; 173: 377-379$

14. Herman D, Stros P, Curila K, et al. Deactivation of implantable cardioverter defibrillators: results of patient surveys. Europace Epub ahead of print 27 February 2013. DOI: 10.1093/europace/eus432.

15. Kuzel A. Sampling in qualitative inquiry. In: Crabtree B and Miller W (eds) Doing Qualitative Research. 2nd ed. Thousand Oaks, CA: Sage Publications, 1999.

16. Rickham P. Human experimentation. Code of ethics of the world medical association. Declaration of Helsinki. Br Med J 1964;18:177.

17. Fluur C, Bolse K, Strömberg A, et al. Patients' experiences of the implantable cardioverter defibrillator (ICD); with a focus on battery replacement and end-of-life issues. Heart \& Lung 2013; 42: 202-207. 
18. Polit DF and Beck CT. Nursing research: generating and assessing evidence for nursing practice. 8th ed. Philadelphia: Lippincott, Williams \& Wilkins, 2008.

19. Willis GB. Cognitive interviewing: a tool for improving questionnaire design. Thousand Oaks, CA, Sage, 2005.

20. Altman DG. Practical statistics for medical research. 1st ed. London: Chapman \& Hall, 1991.

21. Nunnally JC, Bernstein IH. Psychometric theory. 3rd ed. New York, NY: McGrawHill, 1994.

22. Brislin RW. Back-Translation for Cross-Cultural Research. J Cross Cult Psychol 1970; 1: $185-216$.

23. Cha ES, Kim KH and Erlen JA. Translation of scales in cross-cultural research: issues and techniques. $J$ Adv Nurs 2007; 58: 386-395.

24. Marx, RG, Menezes, A, Horovitz, L, Jones, EC, \& Warren, RF. A comparison of two time intervals for test-retest reliability of health status instruments. J Clin Epidemiol 2003; 56: 730-735.

25. Lampert R, Hayes DL, Annas GJ, Farley MA, Goldstein NE, Hamilton RM, Kay GN, Kramer DB, Mueller PS, Padeletti L, Pozuelo L, Schoenfeld MH, Vardas PE, Wiegand DL, Zellner R; American College of Cardiology; American Geriatrics Society; American Academy of Hospice and Palliative Medicine, American Heart Association; European Heart Rhythm Association; Hospice and Palliative Nurses Association. HRS Expert Consensus Statement on the Management of Cardiovascular Implantable Electronic Devices in patients nearing end of life or requesting withdrawal of therapy. Heart Rhythm 2010; 7: 1008-1026.

26. Padeletti L, Arnar DO, Boncinelli L, Brachman J, Camm JA, Daubert JC, Hassam SK, Deliens L, Glikson M, Hayes D, Israel C, Lampert R, Lobban T, Raatikainen P, Siegal 
G, Vardas P; Reviewers:, Kirchhof P, Becker R, Cosio F, Loh P, Cobbe S, Grace A, Morgan J; European Heart Rhythm Association; Heart Rhythm Society. EHRA Expert Consensus Statement on the management of cardiovascular implantable electronic devices in patients nearing end of life or requesting withdrawal of therapy. Europace 2010; 12: 1480-1489. 
Figure 1. Instrument construction process

Phase 1. Instrument development

- Litterature review

- Qualitative study

- Expert discussions and evaluation

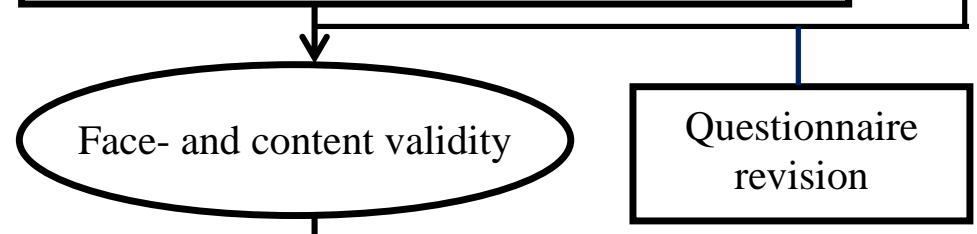

Phase 2. Pilot study, $n=32$

- Questionnaire testing

- Evaluating survey

- Cognitive interviews

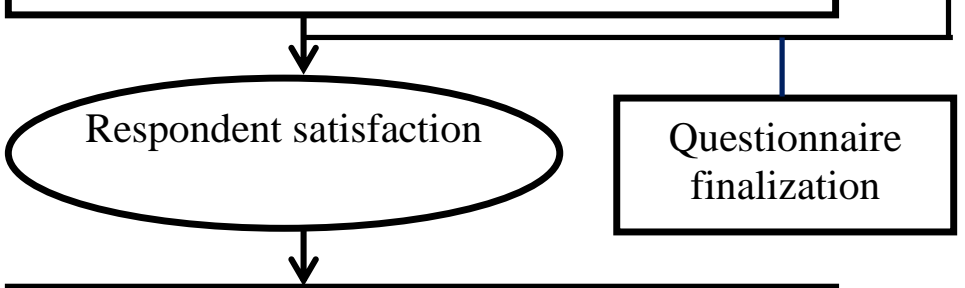

Phase 3. Test- retest study, $n=109$

- Completing final questionnaire twice

- Statistical analyses

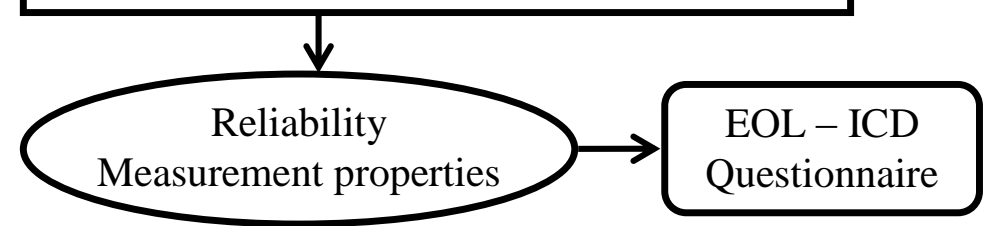


Table 1. Characteristics of the ICD-patients included in the test-retest study $(N=109)$

\begin{tabular}{|c|c|}
\hline Characteristics & \\
\hline Demographic & \\
\hline Age, years & $67.3(+/-13.2)$ \\
\hline Male sex, \% & 80.7 \\
\hline Marital status, married, \% & 75.2 \\
\hline Lower educational level, \% & 38.5 \\
\hline Employment status, working, \% & 31.2 \\
\hline ICD indication & \\
\hline Secondary prevention, \% & 78.0 \\
\hline Resynchronization therapy, CRT-D & \\
\hline Yes, \% & 29.8 \\
\hline Time since implantation, years & $4.85(1-19)$ \\
\hline Generator replacement, yes, $\%$ & 23.9 \\
\hline Experienced a defibrillator shock, yes $\%$ & 43.0 \\
\hline Subjective general health status & $n=108$ \\
\hline Very good or Good, \% & 61.1 \\
\hline Fairly good, \% & 36.1 \\
\hline Poor or Very poor \% & 2.8 \\
\hline VAS score, best possible health & $73.5(+/-18.1)$ \\
\hline Self reported cardiac history & $n=89$ \\
\hline Atrial fibrillation, $\%$ & 54.0 \\
\hline Angina pectoris, $\%$ & 28.2 \\
\hline Myocardial infarction, \% & 36.4 \\
\hline Heart failure, $\%$ & 51.1 \\
\hline
\end{tabular}


Self reported co-morbidities

Diabetes, \%

Hypertension, \%

Renal failure, \%

Stroke, \%

Lung problems, \%

Cancer, \%

ICD = Implantable Cardioverter Defibrillator $\mathrm{n}=95$

21.5

28.4

12.0

5.4

20.0

10.6 


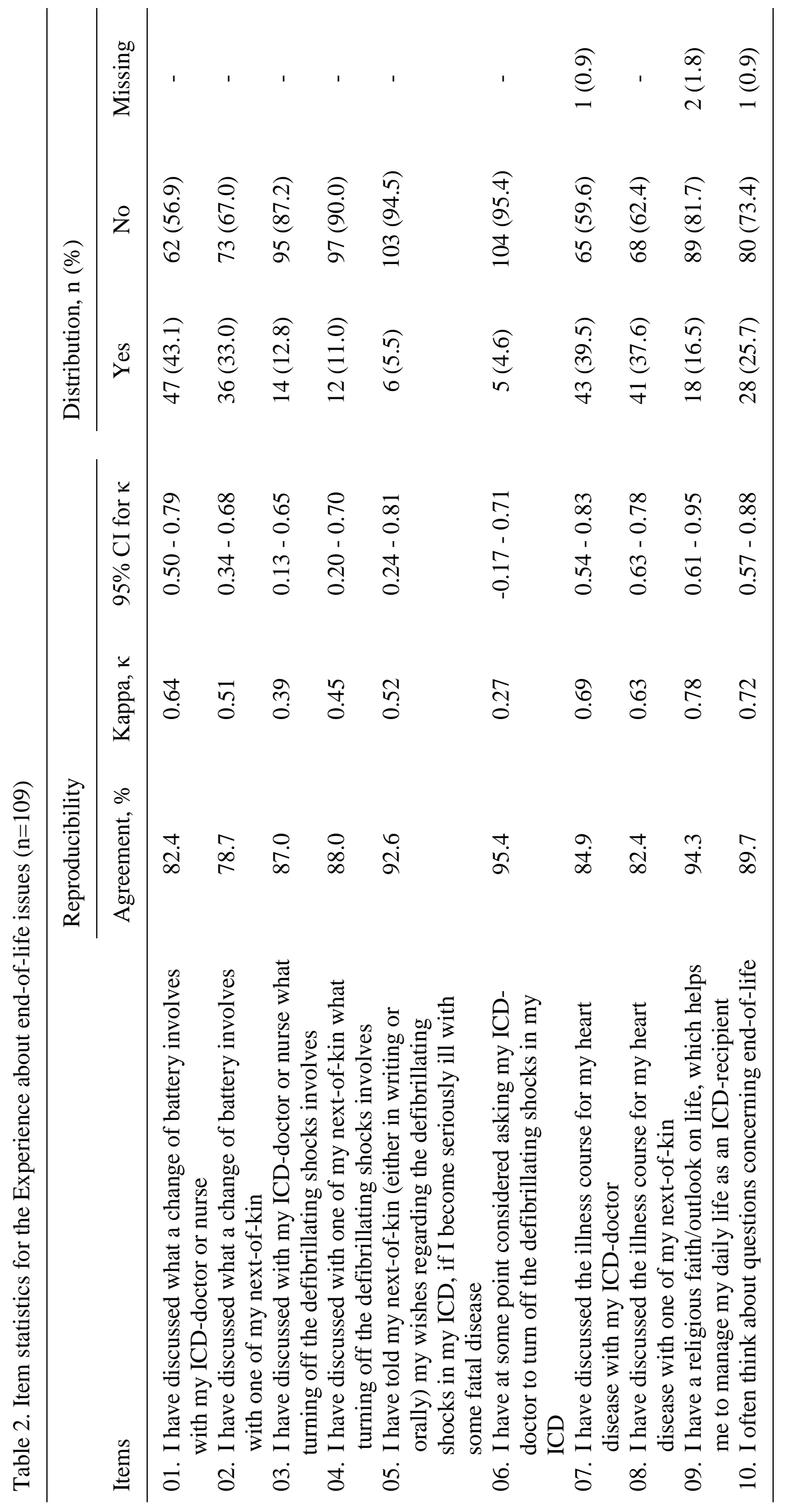




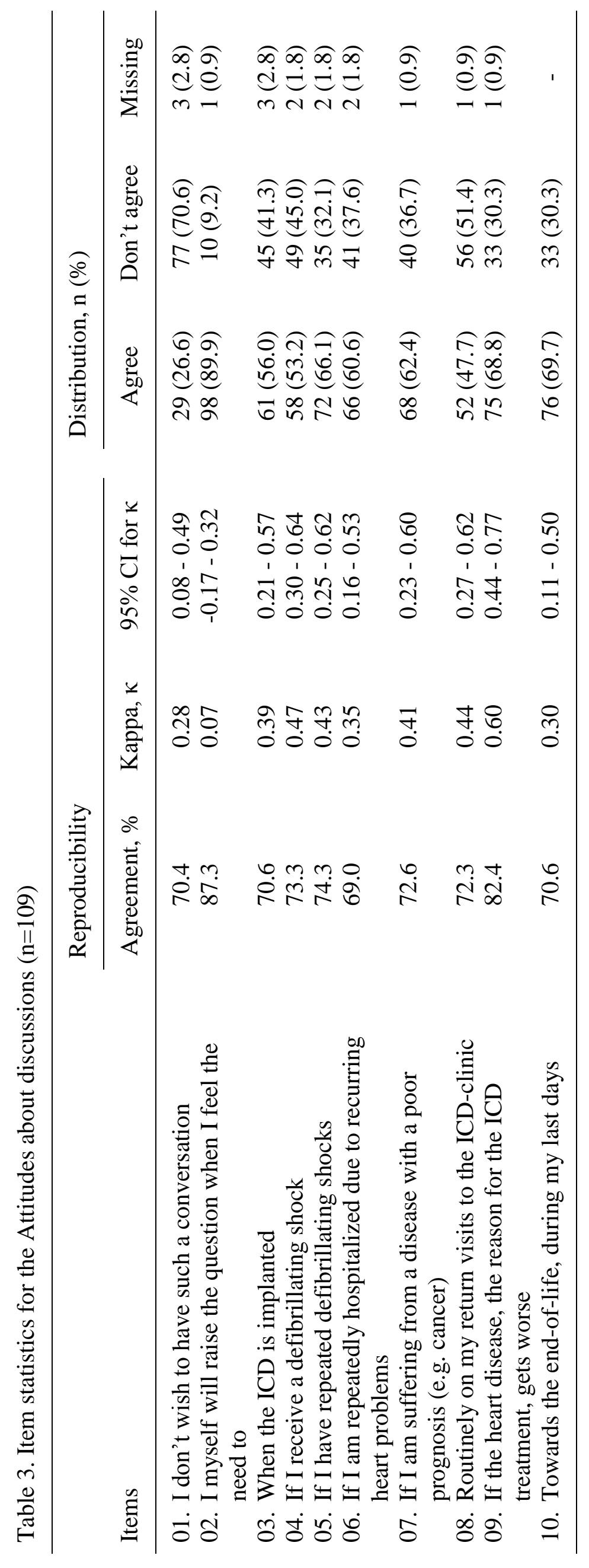




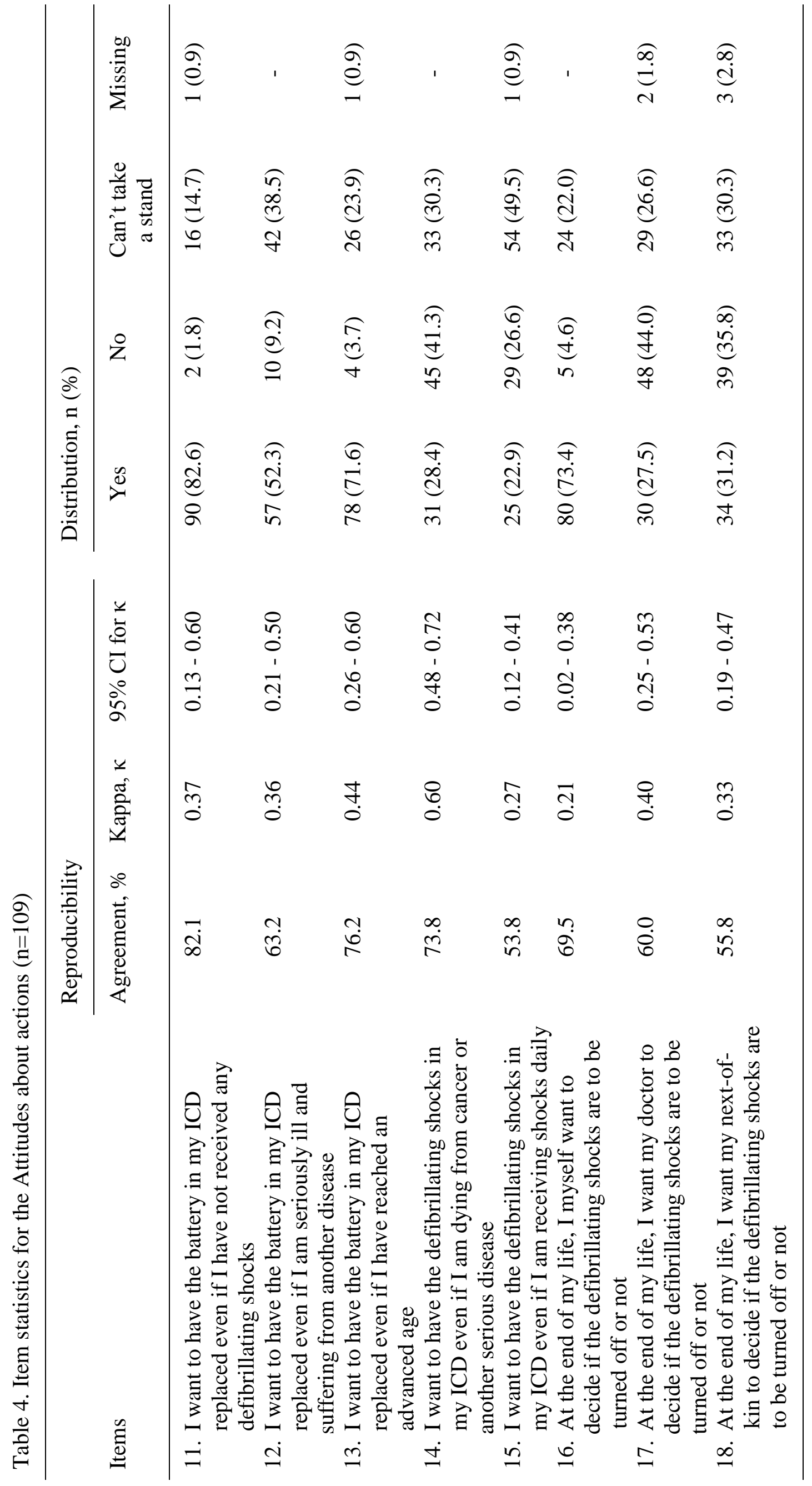




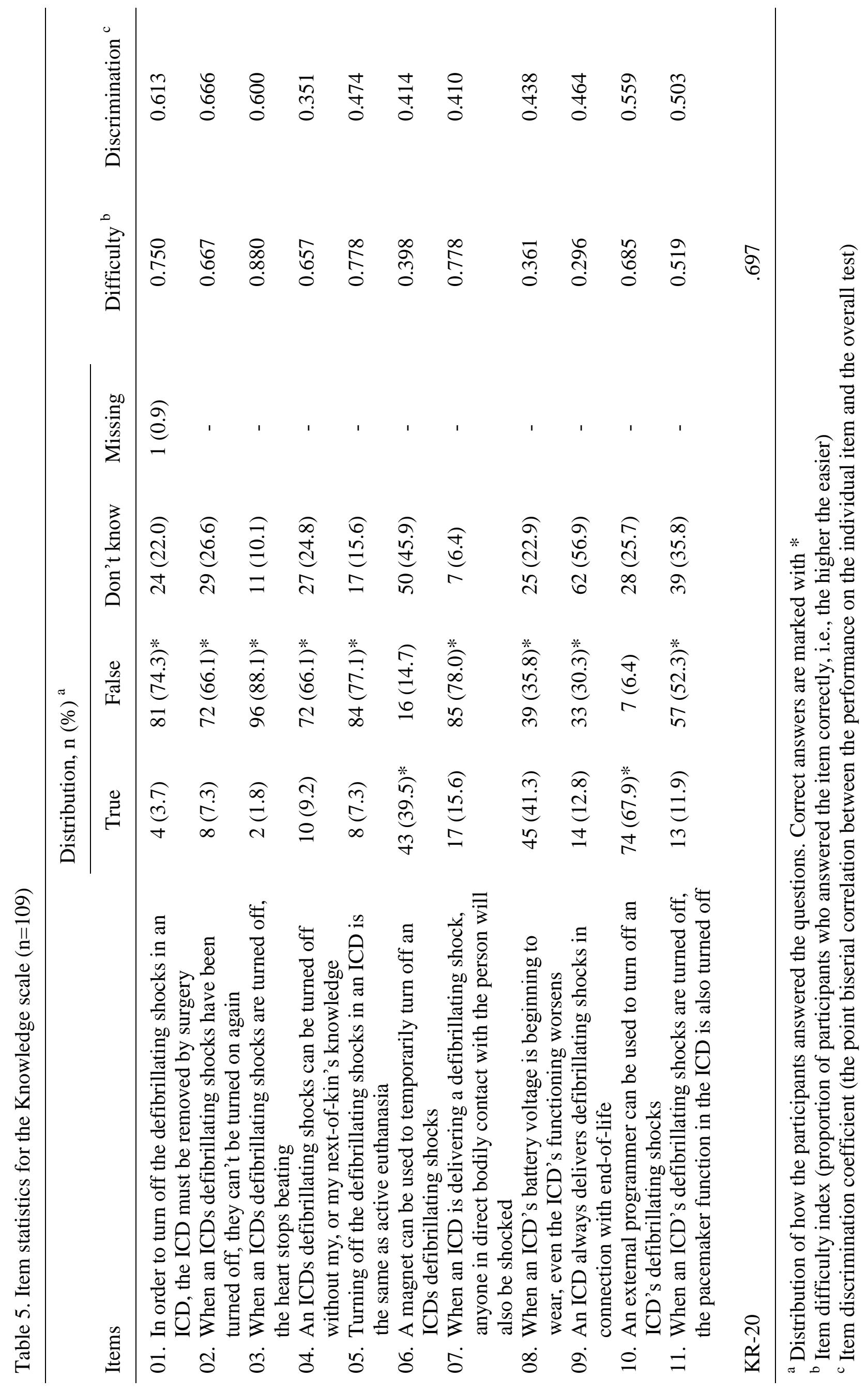




\section{IMPLICATIONS FOR PRACTICE}

EOL-ICDQ can be used as

- a communicating tool for discussing experiences and attitudes about end-of-life

- a reference point for patients' preferences at end-of-life, allowing for continuity of care

- an educational tool for assessing knowledge about ethical aspects, function of the ICD, and practical consequences in end-of-life 